\title{
Divergência genética entre linhagens de matrizes de corte por meio de análise de agrupamento ${ }^{1}$
}

\section{Marcos Yamaki2 ${ }^{2}$, Gilberto Romeiro de Oliveira Menezes 3 ,4, Rafael Bastos Teixeira ${ }^{3,4}$, Leandro Barbosa ${ }^{2}$, André Luis da Costa Paiva ${ }^{2}$, Robledo de Almeida Torres ${ }^{5}$}

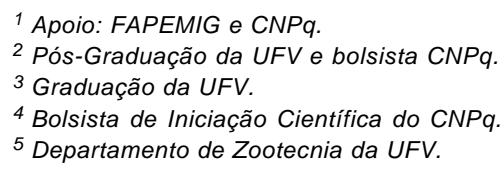

RESUMO - Este trabalho foi realizado com o objetivo de avaliar a divergência genética de três linhagens de matrizes de corte do Programa de Melhoramento Genético da UFV. Foram avaliados dados de 270 aves, 90 de cada linhagem, para estudo das características dias para o primeiro ovo (DPPO), taxa de postura da $22^{\underline{a}}$ à $56^{\underline{a}}$ semana (TP), peso médio individual na

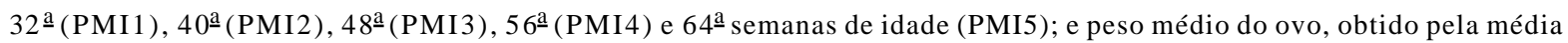

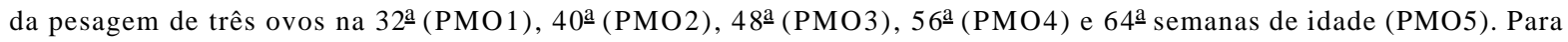
avaliar a divergência, foram utilizados dois métodos: hierárquico do vizinho mais próximo e otimização de Tocher. Pelo método hierárquico do vizinho mais próximo, utilizando-se a distância de Mahalanobis ao quadrado $\left(\mathrm{D}^{2}\right)$ como medida de dissimilaridade, formou-se um único grupo. Pelo método de otimização de Tocher, foram formados dois grupos, comprovando que os dois métodos foram discordantes na avaliação da divergência genética de linhas de aves de corte. As características que apresentaram as contribuições relativas mais expressivas para a divergência foram, respectivamente, PMO1 (25,71\%), DDPO (21,76\%), PMI4 (17,65\%) e PMI2 (13,04\%).

Palavras-chave: distância de Mahalanobis, frango de corte, método de otimização de Toucher, método do vizinho mais próximo

\section{Genetic divergence in meat-type hens using cluster analysis}

\begin{abstract}
Genetic divergence among three lineages of meat-type hens from the Genetic Breeding Program of the Universidade Federal de Viçosa was evaluated for the following traits: days at first egg (DPPO), egg production rate (TP) from $22^{\text {nd }}$ to $56^{\text {th }}$ week, body weight on the $32^{\text {nd }}$ (PMI), on $40^{\text {th }}$ (PMI2), at $48^{\text {th }}$ (PMI3), at 56 ${ }^{\text {th }}$ (PMI4) and at the $64^{\text {th }}$ week (PMI5), egg weight on the $32^{\text {nd }}$ (PMO1), on $40^{\text {th }}$ (PMO2), at $48^{\text {th }}$ (PMO3), at 56 $6^{\text {th }}$ (PMO4) and at the $64^{\text {th }}$ week (PMO5). Traits were measured on 270 hens (90 of each lineage) and two different methods were used to evaluate genetic divergence. For the single linkage hierarchical method, using the squared Mahalanobis distance $\left(\mathrm{D}^{2}\right)$ as the dissimilarity measure, only one single group was formed. When using Tocher's optimization method, two groups were formed, thus indicating a disagreement between the results of the two methods. PMO1 (25.71\%), DDPO $(21.76 \%)$, PMI $(17.65 \%)$ e PMI $2(13.04 \%)$ were the traits with the most expressive relative contributions to genetic divergence among the lineages.
\end{abstract}

Key Words: broiler, Mahalanobis distance, single linkage, Tocher optimization method

\section{Introdução}

A diversidade genética entre um grupo de progenitores tem sido avaliada com o objetivo de identificar as combinações híbridas de maior efeito heterótico e maior heterozigose, de forma que, em suas gerações segregantes, seja obtida maior possibilidade de recuperação de genótipos superiores (Cruz et al., 2004).
A partir do estudo de divergência genética, pode-se avaliar: o grau de divergência entre grupos de genótipos; o comportamento de genótipos em diferentes ambientes; e a superioridade de alguns genótipos sobre outros, com base na combinação linear de características de importância econômica; identificar genótipos divergentes que possam ser utilizados como progenitores em programas de hibridação; e relacionar a divergência genética com a

Este artigo foi recebido em 20/11/2006 e aprovado em 29/10/2007. Correspondências devem ser enviadas para marcosyamaki@hotmail.com 
heterose e/ou diversidade de origem geográfica (Piassi, 1995).

Com o objetivo de melhorar o aproveitamento das vantagens fornecidas pela heterose, a identificação de genótipos divergentes tem sido satisfatoriamente explorada por técnicas de análise multivariada, como a análise de agrupamento, que tem por finalidade reunir, por algum critério de classificação, os progenitores (ou qualquer outro tipo de unidade amostral) em vários grupos, de modo que haja homogeneidade dentro do grupo e heterogeneidade entre grupos. Essa técnica visa dividir um grupo original de observações em vários grupos segundo algum critério de similaridade ou dissimilaridade.

O processo de agrupamento envolve basicamente duas etapas: a primeira é a estimação de uma medida de similaridade (ou dissimilaridade) entre os progenitores e a segunda, a adoção de uma técnica de agrupamento para formação dos grupos. Ainda na segunda etapa, há um processo de estimação de uma medida de similaridade, ou dissimilaridade, entre os progenitores e outra de adoção de uma técnica de agrupamento para formação dos grupos (Cruz et al., 2004).

Estudos de divergência genética são geralmente realizados utilizando-se a distância generalizada de Mahalanobis ao quadrado $\left(D^{2}\right)$ e o método de otimização de Tocher (Rao, 1952) como técnica de agrupamento. Neste método, é estabelecido o critério de manter a distância média intragrupo sempre inferior a qualquer distância intergrupo (Cruz et al., 2004).

Este trabalho foi realizado com o objetivo de avaliar a divergência genética entre três linhagens de matrizes pesadas por meio de análise de agrupamento utilizando-se métodos hierárquicos e de otimização.

\section{Material e Métodos}

Os dados utilizados neste trabalho foram provenientes de três linhagens de matrizes de corte desenvolvidas pela Universidade Federal de Viçosa, obtidas por inseminação artificial, em galpão aberto com gaiolas. Foram avaliadas 270 aves, 90 de cada linhagem, para análise das características: dias para o primeiro ovo (DPPO); taxa de postura da

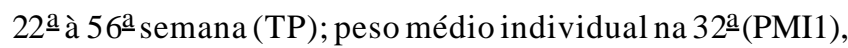

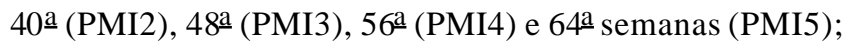
e peso médio do ovo, obtido pela média da pesagem de três

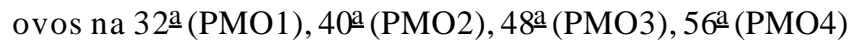
e 64a semanas (PMO5).

As análises de agrupamento foram conduzidas adotando-se a distância generalizada de Mahalanobis ao quadrado $\left(\mathrm{D}^{2}\right)$ como medida de dissimilaridade e empregando-se dois métodos de agrupamento: o hierárquico do vizinho mais próximo e o de otimização de Tocher.

A distância generalizada de Mahalanobis ao quadrado $\left(D^{2}\right)$ entre as linhagens i e i' é determinada por $D_{i i}=\left(\overline{X_{i}}-\overline{X_{i}}\right) R^{-1}\left(\overline{X_{i}}-\overline{X_{i}}\right)$, em que Ré a matriz de co-variância residual; e $\overline{X_{i}}-\overline{X_{i}}$ vetores $\mathrm{p}$-dimensionais de médias das linhagens i e i', respectivamente (Mahalanobis, 1936; Cruz et al., 2004).

No método hierárquico do vizinho mais próximo, as linhagens (linhas de matrizes de corte) foram agrupadas, por meio das menores distâncias $\mathrm{D}^{2}$, por um processo que se repete em vários níveis até que seja estabelecido o dendrograma ou diagrama de árvore. Neste caso, não houve preocupação com o número ótimo de grupos, uma vez que o interesse está na "árvore" e nas ramificações obtidas. As delimitações poderiam ser estabelecidas por um exame visual do dendrograma, no qual se avaliariam pontos de alta mudança de nível, tornando-os em geral delimitadores do número de linhagens para determinado grupo (Cruz et al., 2004).

No método de Tocher, citado por Rao (1952), adota-se o critério de que a média das medidas de dissimilaridade dentro de cada grupo deve ser menor queas distâncias médias entre quaisquer grupos. A decisão de incluir uma linhagem em um grupo foi tomada após as comparações entre o acréscimo no valor médio da distância dentro do grupo e o valor máximo ( $\boldsymbol{\theta})$ da distância encontrada no conjunto das menores distâncias envolvendo cada linhagem.

Identificou-se, inicialmente, o par de linhagens com menor valor de distância $D_{i i}^{2}$ para formar o primeiro grupo quando essa distância não superava o limite estabelecido. Na seqüência, de acordo com o critério adotado, avaliou-se a possibilidade de inclusão da outra linhagem no grupo ou se haveria necessidade de formação de outro grupo, seguindo o mesmo critério. O acréscimo médio no valor da distância intragrupo foi obtido pela razão entre a distância entre a linhagem a ser inserida e o grupo que poderia recebê-la e o número de linhagens do grupo.

Esquematicamente, mostra-se o critério para formação do agrupamento:

$$
\begin{aligned}
& D_{(i j) k}^{2}=D_{i k}^{2}+D_{j k}^{2} \text { ou } D_{(i j k) l}^{2}=D_{(i j) l}^{2}+D_{k l}^{2} \\
& \operatorname{Se} \frac{D^{2}(\text { Grupo }) i}{g} \leq \boldsymbol{\theta} \Rightarrow \text { inclui-se a linhagem no grupo; } \\
& \mathrm{Se} \frac{D^{2}(\text { Grupo }) i}{g}>\boldsymbol{\theta} \Rightarrow \text { não se inclui a linhagem no grupo; }
\end{aligned}
$$

em que: $\theta$ = limite de acréscimo adotado; j, k el = linhagens do grupo; i = linhagem a ser incluída, ou não, no grupo; $\mathrm{g}=$ =úmero de linhagens que formam o grupo que está sendo formado. 
Esse método difere dos hierárquicos porque nele os grupos são formados mutuamente exclusivos ou, sob contexto de teoria de conjuntos, porque subdivide o grupo original em subgrupos não-vazios, cuja interseção é nula e a união reconstitui o conjunto total (Cruz, 1990). Realizou-se a adequação da partição dos grupos por meio da analise discriminante de Anderson. Ao considerarem n populações ou grupos $\Pi_{1}, \ldots, \Pi_{i}, n \geq 2$, em que está associada a cada grupo $\Pi_{i}$ uma distribuição normal multivariada e, ainda, supondo-se a igualdade das matrizes de co-variâncias, as funções discriminantes são obtidas de acordo com a expressão (Anderson, 1958):

$$
\left.D_{i}(\mathrm{X})=\left(\Sigma^{-1} \overline{X_{i}}\right)^{t}\right)_{\sim}^{\mathrm{X}}-\frac{1}{2}\left(\Sigma^{-1} \overline{X_{i}}\right)^{t} \overline{X_{i}}+\ln \left(p_{i}\right)
$$

em que: $D_{i}(\underset{\sim}{\mathrm{X}})=$ escore de classificação do grupo $\mathrm{i} ; \Sigma^{-1}=$ inversa da matriz de co-variâncias; $\overline{\mathrm{X}}=$ vetor de médias do grupo $\mathrm{i} ; \underset{\sim}{\mathrm{X}}=$ vetor de observações da linhagem que se deseja classificar; e $p_{i}=$ probabilidade a priori de que uma linhagem pertença ao grupo i.

Os valores de $p_{i}$ empregados foram $\mathrm{p}_{1}=\mathrm{p}_{2}=1 / 2$, o que significa a mesma probabilidade de classificação de uma linhagem em qualquer um dos dois grupos.

A nova linhagem é classificada ao grupo para o qual tem maior escore de classificação, ou seja, a linhagem desconhecida $(\mathrm{X})$ será classificada no grupo $\prod_{i}$ se, somente se, $D_{i}(\underset{\sim}{\mathrm{X}})=\operatorname{máx}\left[D_{1} \underset{\sim}{\mathrm{X}}, D_{2} \underset{\sim}{\mathrm{X}}, \ldots, D_{n} \underset{\sim}{\mathrm{X}}\right]$.

A importância relativa das características para a divergência genética foi feita conforme metodologia de Singh (1981).

As análises descritas foram realizadas utilizando-se o programa computacional GENES (Cruz, 2001).

\section{Resultados e Discussão}

Entre as linhagens 1 e $2\left(D^{2}=0,015819\right)$, o valor de $D^{2}$ é máximo e o valor mínimo é observado entre as linhagens 1 e $3\left(D^{2}=0,010844\right)$, comprovando que as linhagens 1 e 3 são as mais similares e que as linhagens 1 e 2 são as mais divergentes (Tabela 1).

Segundo Silveira Neto (1986), extrapolações de estudos dessa natureza não são válidas, uma vez que valores das medidas de dissimilaridade são específicos para o estudo em questão e não podem ser comparados a amostras e populações que não foram envolvidas nas análises.

A análise de dendrograma permite a verificação do grau de similaridade entre progenitores, entre progenitores e grupos similares ou entre dois grupos distintos. Neste
Tabela 1 - Distâncias generalizadas de Mahalanobis ao quadrado $\left(D^{2}\right)$ entre as linhagens

\begin{tabular}{llcc}
\hline Linhagem & 1 & 2 & 3 \\
\hline 1 & 0 & 0,015819 & 0,010844 \\
2 & & 0 & 0,012381 \\
3 & & & 0 \\
\hline
\end{tabular}

caso, o estabelecimento dos grupos é feito de forma subjetiva com base nas mudanças acentuadas de níveis associadas ao conhecimento prévio do pesquisador acerca dos progenitores (linhagens de aves de corte) avaliados.Desse modo, pode ser formado um único grupo, visto o alto nível de similaridade (próximo de $90 \%$ ) pelo método do vizinho mais próximo (Figura 1).

Na etapa final do processo de agrupamento, é necessário julgar a adequação dos resultados, pois, na formação do dendrograma, pode ocorrer considerável simplificação das informações originais e podem ser geradas algumas distorções sobre o padrão de similaridade entre os genótipos estudados. Assim, o uso de dendrogramas necessitaria de avaliação mais cautelosa, pois a inferência quanto ao número de grupos ótimos pode ser de difícil visualização, embora os resultados sejam usualmente representados por diagramas de "árvore", facilmente interpretáveis.

Segundo Cruz et al. (2004), comumente são utilizados vários números de grupos e, por algum critério de otimização, seleciona-se o mais conveniente; um método amplamente utilizado no melhoramento genético e com boa freqüência na literatura é o método de Tocher. Neste estudo, observou-se a formação de dois grupos (Tabela 2): o grupo I foi composto pelas linhagens 1 e 3, e o grupo II pela linhagem 2, enquanto, pelo método do vizinho mais próximo, formou-se apenas um grupo, como descrito anteriormente.

Um reexame é necessário para os resultados obtidos pelos métodos de agrupamento da matriz de dados para avaliação da adequação da partição; neste caso, utiliza-se comumente a análise discriminante. A consistência do padrão do agrupamento obtido pelo método de otimização pode ser avaliada aplicando-se análise discriminante aos dados obtidos, geralmente baseada na metodologia de Anderson. Neste estudo, esta metodologia confirmou a partição em dois grupos, o grupo I formado pelas linhagens 1 e 3 e o grupo II formado pela linhagem 2.

Considerando as características relacionadas diretamente à produtividade das aves, aquelas do grupo II (linhagem2) apresentaram maior peso médio individual em todos os períodos, característica altamente desejável para frangos de corte. Entretanto, apresentaram menor taxa de postura e menor peso médio do ovo, apesar de terem sido 


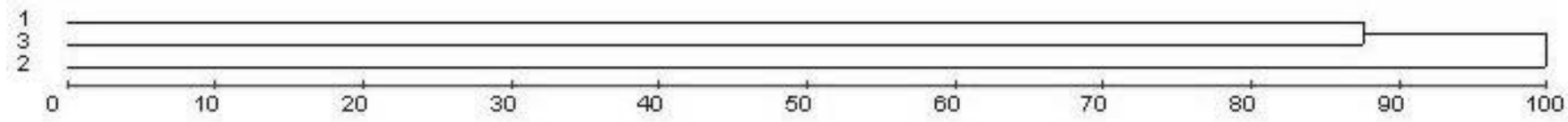

Figura 1 - Dendrograma obtido pelo método do vizinho mais próximo, com a distância $D^{2}$ (expressa em porcentagem), entre as linhagens avaliadas.

Tabela 2 - Médias das características, em cada grupo: grupo I formado pelas linhagens 1 e 3 e grupo II formado pela linhagem 2 pelo método de otimização de Tocher

\begin{tabular}{lcc}
\hline Característica & Grupo I & Grupo II \\
\hline TP (\%) & 74,27 & 71,15 \\
DDPO (dias) & 172,40 & 165,51 \\
PMO1 (g) & 56,45 & 54,24 \\
PMO2 (g) & 61,07 & 60,83 \\
PMO3 (g) & 63,69 & 62,49 \\
PMO4 (g) & 65,21 & 64,14 \\
PMO5 (g) & 68,58 & 67,62 \\
PMI1 (kg) & 2,294 & 2,377 \\
PMI2 (kg) & 3,804 & 4,042 \\
PMI3 (kg) & 4,169 & 4,341 \\
PMI4 (kg) & 4,067 & 4,327 \\
PMI5 (kg) & 4,034 & 4,182 \\
\hline 1 TP - taxa de postura da 22a à 56a semana, DPPO - dias para o primeiro \\
ovo, PMO1 - peso médio do ovo na 32a semana, PMO2 - peso médio do \\
ovo na 40a semana, PMO3 - peso médio do ovo na 48a semana, PMO4 \\
- peso médio do ovo na 56a semana e PMO5 - peso médio do ovo na 64a \\
semana, PMl1 - peso médio individual na 32a semana, PMI2 - peso médio \\
individual na 40a semana, PMI3 - peso médio individual na 48a semana, \\
PMl4 - peso médio individual na 56a semana, PMI5 - peso médio individual \\
na 64a semana
\end{tabular}
na 64 a semana.

mais precoce em detrimento ao maior peso médio individual (Tabela 2), em comparação ao grupo I (linhagens 1 e 3).

O método de otimização de Tocher e o método hierárquico do vizinho mais próximo foram discordantes na partição dos grupos, o que está de acordo com os resultados encontrados por Sakaguti et al. (1996).

As características que apresentaram as contribuições relativas mais expressivas para a divergência foram, respectivamente, DDPO (21,76\%), PMO1 (25,71\%), PMI2(13,04\%) e PMI4 (17,65\%) (Tabela 3). O número de características avaliadas na seleção de genótipos superiores é de grande relevância em um programa de melhoramento genético, uma vez que está diretamente relacionado à eficiência e à viabilidade deste programa. Quanto maior o número de características envolvidas em uma avaliação, menor a eficiência de ganho e maiores o custo e o tempo de execução do programa de melhoramento. Portanto, características com pouca variação entre os genótipos contribuem pouco para a divergência genética e podem ser eliminadas.
Tabela 3 - Importância relativa das características (S) para o estudo da divergência genética em três linhagens de matrizes de corte

\begin{tabular}{lcc}
\hline Variável $^{1}$ & $\mathrm{~S}_{\mathrm{j}}$ & $\mathrm{S}_{\mathrm{j}} \%$ \\
\hline TP $(\%)$ & 0,000675 & 1,53 \\
DDPO (dias) & 0,009606 & $\mathbf{2 1 , 7 6}$ \\
PMO1 (g) & 0,011352 & $\mathbf{2 5 , 7 1}$ \\
PMO2 (g) & 0,001205 & 2,73 \\
PMO3 (g) & 0,001957 & 4,43 \\
PMO4 (g) & 0,000952 & 2,16 \\
PMO5 (g) & 0,000600 & 1,36 \\
PMI1 (kg) & 0,000585 & 1,32 \\
PMI2 (kg) & 0,005757 & $\mathbf{1 3 , 0 4}$ \\
PMI3 (kg) & 0,001703 & 3,86 \\
PMI4 (kg) & 0,007792 & $\mathbf{1 7 , 6 5}$ \\
PMI5 (kg) & 0,001970 & 4,46 \\
\hline
\end{tabular}

1 TP - taxa de postura da $22 \underline{a}$ à 56 a semana, DPPO - dias para o primeiro ovo, PMO1 - peso médio do ovo na 32 a semana, PMO2 - peso médio do ovo na 40a semana, PMO3 - peso médio do ovo na 48a semana, PMO4 - peso médio do ovo na 56a semana e PMO5 - peso médio do ovo na $64^{2}$ semana, PMI1 - peso médio individual na 32ª semana, PMI2 - peso médio individual na 40 a semana, PMI3 - peso médio individual na $48^{\mathrm{a}}$ semana, PMI4 - peso médio individual na 56a semana, PMI5 - peso médio individual na 64a semana.

\section{Conclusões}

Apesar de discordantes na formação de grupos da avaliação da divergência de linhagens de corte, o método hierárquico do vizinho mais próximo e o de otimização de Tocher contribuem para a interpretação da divergência de grupos genéticos. A partir da obtenção dos grupos genéticos formados pelo método de otimização de Tocher, estratégias de cruzamentos entre as linhagens podem ser traçadas para maximização da heterose e da complementaridade das características desejáveis em frangos de corte.

\section{Literatura Citada}

ANDERSON, T.W. An introduction to multivariate statistical analysis. New York: John Wiley \& Sons, 1958. 374p.

CRUZ, C.D. Aplicação de algumas técnicas multivariadas no melhoramento de plantas. Piracicaba: Escola Superior de Agricultura Luiz de Queiroz, 1990. 188p. Dissertação (Doutorado em Genética e Melhoramento) - Escola Superior de Agricultura Luiz de Queiroz, 1990. 
CRUZ, C.D. Programa Genes: versão Windows; aplicativo computacional em genética e estatística. Viçosa, MG: Universidade Federal de Viçosa, 2001. 648p.

CRUZ C.D.; REGAZZI A.J.; CARNEIRO P.C.S. Modelos biométricos aplicados ao melhoramento genético. 3.ed. Viçosa, MG: Universidade Federal de Viçosa, 2004. $480 \mathrm{p}$.

MAHALANOBIS, P.C. On the generalized distance in statistics. Proceedings of Natural Institute of Sciences, v.2, p.49$55,1936$.

PIASSI M.; SILVA M.A, REGAZZI A.J. et al. Estudo da divergência genética entre oito grupos de aves de postura, por meio de técnicas de análise multivariada. Revista Brasileira de Zootecnia, v.24, p.715-727, 1995.

RAO C.R. Advanced statistical methods in biometric research New York: John Wiley \& Sons; 1952. 390p.

SAKAGUTI E.; SILVA M.A.; REGAZZI A.J. et al. Análise de divergência genética entre nove grupos genéticos de coelhos. Revista Brasileira de Zootecnia, v.25, p.647-660, 1996.

SINGH, D. The relative importance of characters affecting genetic divergence. The Indian Journal of Genetic and Plant Breeding, v.41, p.237-245, 1981.

SILVEIRA NETO, S. Análise genética. In: ALVES, S.B. (Ed.) Controle microbiano de insetos. São Paulo: Manole, 1986. p.384-407. 